\title{
A NOTE ON ZERO-SETS OF FRACTIONAL SOBOLEV FUNCTIONS WITH NEGATIVE POWER OF INTEGRABILITY
}

\author{
ARMIN SCHIKORRA
}

(Communicated by Jeremy Tyson)

\begin{abstract}
We extend a Poincaré-type inequality for functions with large zero-sets by Jiang and Lin to fractional Sobolev spaces. As a consequence, we obtain a Hausdorff dimension estimate on the size of zero-sets for fractional Sobolev functions whose inverse is integrable. Also, for a suboptimal Hausdorff dimension estimate, we give a completely elementary proof based on a pointwise Poincaré-style inequality.
\end{abstract}

\section{INTRODUCTION}

Let $\Omega \subset \mathbb{R}^{n}$ be an open set. For functions $u: \Omega \rightarrow \mathbb{R}^{n}$ we are interested in the size of the zero set $\Sigma$,

$$
\Sigma:=\left\{x \in \Omega: \quad \lim _{r \rightarrow 0} f_{B_{r}(x)}|f|=0\right\},
$$

under the condition that for some $\alpha>0$,

$$
\int_{\Omega}|f|^{-\alpha}<\infty
$$

Here and henceforth, for a measurable set $A \subset \mathbb{R}^{n}$ we denote the mean value integral

$$
f_{A} f \equiv(f)_{A}:=|A|^{-1} \int_{A} f .
$$

In [8] Jiang and Lin showed that if $f \in W^{1, p}(\Omega)$, then

$$
\mathcal{H}^{s}(\Sigma)=0 \quad \text { where } s=\max \left\{0, n-\frac{p \alpha}{p+\alpha}\right\} .
$$

They were motivated by the analysis of rupture sets of thin films, which is described by a singular elliptic equation. We do not go into the details of this; instead, for applications, we refer to, e.g., 2, 3, 6, 7,.

In this note, we extend Jiang and Lin's result to fractional Sobolev spaces and obtain

Theorem 1.1. For $\sigma \in(0,1]$ and for any $f \in W^{\sigma, p}(\Omega)$ satisfying (1.1), $\mathcal{H}^{s}(\Sigma)=0$, where $s=\max \left\{0, n-\sigma \frac{p \alpha}{p+\alpha}\right\}$.

Here, we use the following definitions for the (fractional) Sobolev space. For more on these we refer to, e.g., [1,4,10,

Received by the editors July 19, 2013.

2010 Mathematics Subject Classification. Primary 49Q15; Secondary 46E35.

The author was supported by DAAD fellowship D/12/40670.

(C) 2014 American Mathematical Society Reverts to public domain 28 years from publication 
Definition 1.2. The homogeneous $W^{\sigma, p}$-norms are defined as follows:

$$
[f]_{\dot{W}^{1, p}(\Omega)}:=\|\nabla f\|_{L^{p}(\Omega)} .
$$

For $\sigma \in(0,1)$ we define the Slobodeckij-norm,

$$
[f]_{\dot{W}^{\sigma, p}(\Omega)}:= \begin{cases}\left(\int_{\Omega} \int_{\Omega}\left(\frac{|f(x)-f(y)|}{|x-y|^{\sigma}}\right)^{p} \frac{d x d y}{|x-y|^{n}}\right)^{\frac{1}{p}} & \text { if } p \in[1, \infty), \\ \sup _{x \neq y} \frac{|f(x)-f(y)|}{|x-y|^{\sigma}} & \text { if } p=\infty .\end{cases}
$$

The respective Sobolev space $W^{\sigma, p}, \sigma \in(0,1], p \in[1, \infty]$ is then the collection of functions $f: \Omega \rightarrow \mathbb{R}$ with finite Sobolev norms $\|f\|_{W^{\alpha, p}(\Omega)}$,

$$
\|f\|_{W^{\alpha, p}(\Omega)}:=\|f\|_{L^{p}(\Omega)}+[f]_{\dot{W}^{\alpha, p}(\Omega)} .
$$

To prove Theorem 1.1, the case $p \leq n / \sigma$ is the relevant one, since for the other cases we can use the embedding into the Hölder spaces; see 8 . We have the following extension to fractional Sobolev spaces of a Poincaré-type inequality from [8].

Theorem 1.3. For any $\theta>0, \sigma \in(0,1], p \in(1, n / \sigma], s \in(n-\sigma p, n]$, there is a constant $C>0$ such that the following holds for any $R>0$ :

Let $B_{R}$ be any ball in $\mathbb{R}^{n}$ with radius $R, f \in W^{\sigma, p}\left(B_{R}\right)$ and assume that there is a closed set $T \subset B_{R}$ such that

$$
\begin{gathered}
T \subset\left\{x \in B_{R}: \limsup _{r \rightarrow 0} \int_{B_{r}}|f|=0\right\}, \\
\mathcal{H}^{s}(T)>\frac{1}{\theta} R^{s},
\end{gathered}
$$

and for any ball $B_{r}$ with some radius $r>0$,

$$
\mathcal{H}^{s}\left(T \cap B_{r}\right) \leq \theta r^{s} .
$$

Then,

$$
\|f\|_{L^{p}\left(B_{R}\right)} \leq C R^{\sigma}[f]_{\dot{W}^{\sigma, p}\left(B_{R}\right)} .
$$

In [8] this was proven for the classical Sobolev space $W^{1, p}$, using an argument based on the $p$-Laplace equation with measures and the Wolff potential. Our argument, on the other hand, is completely elementary and adapts the classical blow-up proof of the Poincaré inequality; see Section 2 .

Once Theorem 1.3 is established, one can follow the arguments in 8 to obtain Theorem 1.1. These rely heavily on the theory of Sousslin sets, 9], to find the closed set $T \subset \Sigma$ with the condition (1.2) and (1.3) satisfied. Those arguments are by no means elementary, but we were unable to remove them in order to show that $\mathcal{H}^{s}(\Sigma)=0$. However, if one is satisfied in showing that $\mathcal{H}^{t}(\Sigma)=0$ for any $t>s$, then there is a completely elementary argument, the details of which we will present in Section 3 . There, we prove the following "pointwise" Poincaré-style inequality, from which the suboptimal Hausdorff dimension estimate easily follows; see Corollary 3.1 
Lemma 1.4. For any $\varepsilon>0, p \in[1, \infty)$, there exists $C>0$, such that the following holds. Let $f \in L_{\text {loc }}^{p}$, and assume $x \in \mathbb{R}^{n}$, such that

$$
\lim _{r \rightarrow 0} f_{B_{r}(x)}|f|=0
$$

Then for any $R>0$, there exists $\rho \in(0, R)$ such that

$$
\int_{B_{\rho}(x)}|f|^{p} \leq C\left(\frac{R}{\rho}\right)^{\varepsilon} \int_{B_{\rho}(x)}|| f\left|-(|f|)_{B_{\rho}}\right|^{p} .
$$

\section{Poincaré inequality: Proof of Theorem 1.3}

By a scaling argument, Theorem 1.3 follows from Lemma 2.1.

Lemma 2.1. For any $\theta>0, \sigma \in(0,1], p \in(1, n / \sigma], s \in(n-\sigma p, n]$, there is a constant $C>0$ such that the following holds:

Let $f \in W^{\sigma, p}\left(B_{1},[0, \infty)\right)$ and assume that there is a closed set $T \subset B_{1}$ such that

$$
T \subset\left\{x \in B_{1}: \quad \limsup _{r \rightarrow 0} f_{B_{r}} f=0\right\}
$$

and

as well as

$$
\mathcal{H}^{s}(T)>\frac{1}{\theta}
$$

$\mathcal{H}^{s}\left(T \cap B_{r}\right) \leq \theta r^{s} \quad$ for any ball $B_{r}$ with radius $r>0$.

Then,

$$
\|f\|_{L^{p}\left(B_{1}\right)} \leq C[f]_{\dot{W}^{\sigma, p}\left(B_{1}\right)} .
$$

Proof. We proceed by the usual blow-up proof of the Poincaré inequality: Assume the claim is false, and that for fixed $\theta, p, s, \sigma$ for any $k \in \mathbb{N}$ there are $f_{k} \in W^{\sigma, p}\left(B_{1},[0, \infty)\right)$ such that

$$
\begin{gathered}
T_{k} \subset\left\{x \in B_{1}: \quad \limsup _{r \rightarrow 0} f_{B_{r}} f_{k}=0\right\}, \\
\mathcal{H}^{s}\left(T_{k}\right)>\frac{1}{\theta}, \quad \mathcal{H}^{s}\left(T_{k} \cap B_{r}\right) \leq \theta r^{s} \forall B_{r},
\end{gathered}
$$

and

$$
\left\|f_{k}\right\|_{L^{p}\left(B_{1}\right)}>k\left[f_{k}\right]_{\dot{W}^{\sigma, p}\left(B_{1}\right)} .
$$

Replacing $f_{k}$ by $\frac{f_{k}}{\left\|f_{k}\right\|_{p}}$ (note that this does not change the definition and size of $T_{k}$ ), we can assume w.l.o.g.

$$
\left\|f_{k}\right\|_{L^{p}} \equiv 1
$$

and

$$
\left[f_{k}\right]_{\dot{W}^{\sigma, p}\left(B_{1}\right)} \stackrel{k \rightarrow \infty}{\longrightarrow} 0 .
$$

In particular, $f_{k}$ is uniformly bounded in $W^{\sigma, p}$, and by the Rellich-Kondrachov theorem, up to taking a subsequence, $f_{k}$ converges strongly in $L^{p}$, and weakly in $W^{\sigma, p}$ to some $f \in W^{\sigma, p}$, with $[f]_{W^{\sigma, p}\left(B_{1}\right)} \equiv 0,\|f\|_{L^{p}}=1$. Thus,

$$
f \equiv\left|B_{1}\right|^{-\frac{1}{p}}
$$


and setting $g_{k}:=\left|B_{1}\right|^{\frac{1}{p}} f_{k}$, we have found a sequence such that

and

$$
\begin{gathered}
g_{k} \rightarrow 1 \text { in } W^{\sigma, p}\left(B_{1}\right), \\
\mathcal{H}^{s}\left(T_{k}\right)>\frac{1}{\theta},
\end{gathered}
$$

$$
\mathcal{H}^{s}\left(T_{k} \cap B_{r}\right) \leq \theta r^{s} \quad \text { for any ball } B_{r} .
$$

This is a contradiction to Lemma 2.2

We used the following lemma, which essentially quantifies the intuition, that a function approximating 1 in $W^{\sigma, p}$ cannot be zero on a large set.

Lemma 2.2. Let $\sigma \in(0,1], s \in(n-\sigma p, n], f_{k} \in W^{\sigma, p}\left(B_{1},[0, \infty)\right)$, and assume that

$$
\left\|f_{k}-1\right\|_{W^{\sigma, p}\left(B_{1}\right)} \stackrel{k \rightarrow \infty}{\longrightarrow} 0 .
$$

Then, for any $T_{k} \subset B_{1}$ closed and

$$
T_{k} \subset\left\{x \in B_{1}: \quad \limsup _{r \rightarrow 0} \int_{B_{r}} f_{k}=0\right\},
$$

as well as for some $\theta>0$,

$$
\mathcal{H}^{s}\left(T_{k} \cap B_{r}\right) \leq \theta r^{s} \quad \text { for any } B_{r}, \text { for all } k
$$

we have

$$
\lim _{k \rightarrow \infty} \mathcal{H}^{s}\left(T_{k}\right)=0 .
$$

Proof. By the subsequence principle, it suffices to show

$$
\liminf _{k \rightarrow \infty} \mathcal{H}^{s}\left(T_{k}\right)=0 .
$$

By extension, we also can assume that $f_{k}-1 \rightarrow 0$ in $W^{\sigma, p}\left(\mathbb{R}^{n}\right)$, and $f_{k} \equiv 1$ on $\mathbb{R}^{n} \backslash B_{2}$.

On the one hand, we have

$$
\left[f_{k}\right]_{\dot{W}^{\sigma, p}\left(\mathbb{R}^{n}\right)} \stackrel{k \rightarrow \infty}{\longrightarrow} 0 .
$$

On the other hand, up to picking a subsequence, we can assume the existence of $R_{k} \in(0,1)$, for $k \in \mathbb{N}$, and $\lim _{k \rightarrow \infty} R_{k}=0$, such that

$$
\inf _{r>R_{k}, x \in B_{1}} f_{B_{r}(x)} f_{k} \geq \frac{9}{10} .
$$

Since for any point $x \in T_{k}$ we have that $\lim _{t \rightarrow 0} f_{B_{r}} f_{k}(x)=0$, we expect the average (fractional) gradient around $x$ to be fairly large. More precisely, we have the following

Claim. There is a uniform constant $c_{s, \sigma, p}>0$, such that the following holds: For any $x \in T_{k}$, there exists $\rho=\rho_{k, x} \in\left(0, R_{k}\right)$ such that

$$
c_{s, \sigma, p} \rho^{s} \leq \rho^{-\sigma p} \int_{B_{\rho}}\left|f_{k}-\left(f_{k}\right)_{B_{\rho}}\right|^{p} \leq C\left[f_{k}\right]_{\dot{W}^{\sigma, p}\left(B_{\rho}\right)}^{p} .
$$

Of course, we only have to show the first inequality; the second inequality is the classical Poincaré inequality. 
For the proof let us write $f$ instead of $f_{k}$. Then, since for $x \in T$,

$$
\lim _{l \rightarrow \infty} \int_{B_{2^{-l-1} R_{k}(x)}} f=0
$$

we have that

$$
\begin{aligned}
\frac{9}{10} & \leq \sum_{l=0}^{\infty}\left(\underset{B_{2}-l_{R_{k}}(x)}{f} f-\underset{B_{2}-l-1_{R_{k}}(x)}{f} f\right) \\
& \leq C \sum_{l=0}^{\infty}\left(\left(2^{-l} R_{k}\right)^{-n} \int_{B_{2^{-l} R_{k}}}\left|f-(f)_{B_{2-l} R_{k}}\right|\right) .
\end{aligned}
$$

Consequently, for any $\varepsilon>0$, there has to be some $c_{\varepsilon}>0$ and some $l \in \mathbb{N}$ such that

$$
\left(\left(2^{-l} R_{k}\right)^{-n} \int_{B_{2^{-l} R_{k}}}\left|f-(f)_{B_{2-l} R_{k}}\right|\right) \geq c_{\varepsilon}\left(2^{-l} R_{k}\right)^{\varepsilon}
$$

because if the opposite inequality was true for all $l \in \mathbb{N}$ we would have

$$
\frac{9}{10} \leq C c_{\varepsilon} R_{k}^{\varepsilon} \sum_{l \in \mathbb{N}} 2^{-\varepsilon l} \leq C c_{\varepsilon} \sum_{l \in \mathbb{N}} 2^{-\varepsilon l}
$$

which is false for $c_{\varepsilon}$ small enough.

Thus, for $\rho:=2^{-l} R_{k} \in\left(0, R_{k}\right)$,

$$
\rho^{n-\sigma+\varepsilon} \leq C_{\varepsilon} \rho^{-\sigma} \int_{B_{\rho}}\left|f-(f)_{B_{\rho}}\right| \leq C_{\varepsilon}\left(\rho^{-\sigma p} \int_{B_{\rho}}\left|f-(f)_{B_{\rho}}\right|^{p}\right)^{\frac{1}{p}} \rho^{n-\frac{n}{p}},
$$

that is,

$$
\rho^{n-\sigma p+\varepsilon p} \leq C_{\varepsilon} \rho^{-\sigma p} \int_{B_{\rho}}\left|f-(f)_{B_{\rho}}\right|^{p} .
$$

Setting $\varepsilon=\frac{s-(n-\sigma p)}{p}>0$, we have shown for any $x \in T$ the existence of some $\rho \in\left(0, R_{k}\right)$ satisfying (2.2), and the claim is proven.

For any $k$ we cover $T_{k}$ by the family

$$
\mathcal{F}_{k}:=\left\{B_{\rho}(x), \quad x \in T, B_{\rho}(x) \text { satisfies }(\underline{2.2})\right\} .
$$

Since $T \subset B_{2}$ is closed and bounded, i.e. compact, we can find a finite subfamily still covering all of $T_{k}$, and then using Vitali's (finite) covering theorem, we find a subfamily $\tilde{\mathcal{F}}_{k} \subset \mathcal{F}_{k}$ of disjoint balls $B_{\rho}(x)$, so that the union of the $B_{5 \rho}$ covers all of $T_{k}$. We use this $\tilde{\mathcal{F}}_{k}$ as a cover for an estimate of the Hausdorff measure:

$$
\begin{aligned}
\mathcal{H}^{s}\left(T_{k}\right) & \leq \sum_{B_{\rho} \in \tilde{\mathcal{F}}_{k}} \mathcal{H}^{s}\left(B_{5 \rho} \cap T_{k}\right) \stackrel{\sqrt{2.11})}{\leq} \theta 5^{s} \sum_{B_{\rho} \in \tilde{\mathcal{F}}_{k}} \rho^{s} \\
& \stackrel{(2.2)}{\leq} C_{\theta, s} \sum_{B_{\rho} \in \tilde{\mathcal{F}}_{k}}\left[f_{k}\right]_{\dot{W}^{\sigma, p}\left(B_{\rho}\right)}^{p} \leq C_{\theta, s}\left[f_{k}\right]_{\dot{W}^{\sigma, p}\left(\mathbb{R}^{n}\right)}^{p} \stackrel{k \rightarrow \infty}{\longrightarrow} 0 .
\end{aligned}
$$




\section{An elementary proof For the suboptimal CASE}

We start with the proof of the pointwise inequality, Lemma 1.4

Proof. First, let us show the claim for $p=1$ :

Fix $R, \varepsilon>0, f \in L_{l o c}^{1}$ and assume $x=0$. W.l.o.g., $f \geq 0$. Set

$$
\tau=2^{-n-1}\left(\sum_{l=-\infty}^{0} 2^{\varepsilon l}\right)^{-1} R^{-\varepsilon}
$$

and $C_{\varepsilon}:=R^{-\varepsilon} \tau^{-1}$. Assume by contradiction that the claim was false, i.e. assume that for any $\rho \in(0, R)$,

$$
\int_{B_{\rho}}\left|f-(f)_{B_{\rho}}\right|<\tau \rho^{\varepsilon} \int_{B_{\rho}} f
$$

Then for any $K \in \mathbb{N}$,

$$
\begin{aligned}
f_{B_{\rho}}\left|f-(f)_{B_{\rho}}\right| & <\tau \rho^{\varepsilon} \sum_{k=-K}^{0} f_{B_{2^{k}}} f-f_{B_{2^{k-1} \rho}} f+\tau \rho^{\varepsilon} \underset{B_{2}-K-1 \rho}{f} f \\
& \leq 2^{n} \tau \rho^{\varepsilon} \sum_{k=-K}^{0} f\left|f-(f)_{B_{2^{k}} \rho}\right|+\tau \rho^{\varepsilon} \underset{B_{2^{k}} \rho}{f} f .
\end{aligned}
$$

Setting now for $l \in \mathbb{Z}$,

$$
\begin{gathered}
a_{l}:=\int_{B_{2^{l} R}}\left|f-(f)_{B_{2^{l} R}}\right|, \\
b_{l}:=f_{B_{2^{l} R}} f,
\end{gathered}
$$

the above equation applied to $\rho=2^{l} R$ reads as

$$
a_{l} \leq 2^{n} R^{\varepsilon} \tau 2^{\varepsilon l} \sum_{k=-K}^{0} a_{k+l}+\tau\left(2^{l} R\right)^{\varepsilon} b_{-K+l-1} \quad \text { for any } K \in \mathbb{N}, l \in-\mathbb{N} .
$$

In particular for any $L \in \mathbb{N}$,

$$
\begin{aligned}
\sum_{l=-L}^{0} a_{l} & \leq 2^{n} R^{\varepsilon} \tau \sum_{l=-L}^{0} 2^{\varepsilon l} \sum_{k=-K}^{0} a_{k+l}+\tau R^{\varepsilon} \sum_{l=-L}^{0} 2^{\varepsilon l} b_{-K+l-1} \\
& \leq 2^{n} R^{\varepsilon} \tau \sum_{l=-L}^{0} 2^{\varepsilon l} \sum_{k=-K+l}^{0} a_{k}+\tau R^{\varepsilon}\left(\sup _{j \leq-K} b_{j}\right) \sum_{l=-\infty}^{0} 2^{\varepsilon l} \\
& \leq 2^{n} R^{\varepsilon} \tau \sum_{k=-L-K}^{0} a_{k} \sum_{l=-L}^{k+K} 2^{\varepsilon l}+\tau R^{\varepsilon}\left(\sup _{j \leq-K} b_{j}\right) \sum_{l=-\infty}^{0} 2^{\varepsilon l} \\
& \stackrel{3.1}{\leq} \frac{1}{2} \sum_{k=-L-K}^{0} a_{k}+\frac{1}{2} \sup _{j \leq-K} b_{j} .
\end{aligned}
$$


Under the additional assumption that

$$
\sum_{l=-\infty}^{0} a_{l}<\infty
$$

letting $L, K \rightarrow \infty$, using that by (1.4) we have $\lim _{l \rightarrow \infty} b_{l}=0$, the above estimate implies that $a_{k}=0$ for all $k \leq 0$. This means that $f$ is a constant on $B_{R}$, and in particular by (1.4), $f$ is constantly zero in $B_{R}$. This contradicts the strict inequality (3.2).

To see (3.3), fix $K \in \mathbb{N}$ such that $\sup _{j \leq-K} b_{j} \leq 2$. Then for

$$
c_{L}:=\sum_{l=-L}^{0} a_{l},
$$

the above estimate becomes

$$
c_{L} \leq \frac{1}{2} c_{L+K}+1 \quad \text { for any } L \in \mathbb{N} .
$$

In particular, for any $i \in \mathbb{N}$,

$$
c_{L+i K} \leq 2^{-i} c_{L}+\sum_{j=0}^{i} 2^{-j} .
$$

Since $c_{i}$ is monotonically increasing,

$$
\sup _{i \geq L+K} c_{i} \leq c_{L}+\sum_{j=0}^{\infty} 2^{-j}<\infty
$$

This proves Lemma 1.4 for $p=1$.

If $p>1$, we apply this to $f^{p}$, and obtain

$$
\int_{B_{\rho}(x)} f^{p} \leq C\left(\frac{R}{\rho}\right)^{\varepsilon} \int_{B_{\rho}(x)}\left|f^{p}-\left(f^{p}\right)_{B_{\rho}}\right| .
$$

We now need the following estimate, which holds for any $p \in[1, \infty)$, and $\delta \in(0,1)$ :

$$
|| a-\left.b\right|^{p}-|a|^{p}-\left.|b|^{p}|\leq \delta| a\right|^{p}+\frac{C_{p}}{\delta^{p}}|b|^{p} .
$$

Since $B_{\rho}$ is fixed, let us write $(f)$ for $(f)_{B_{\rho}}$. First, for any $\delta \in(0,1)$,

$$
\left|f^{p}-\left(f^{p}\right)\right| \leq|f-(f)|^{p}+\left|(f)^{p}-\left(f^{p}\right)\right|+\frac{C}{\delta^{p}}|f-(f)|^{p}+\delta(f)^{p} .
$$

Plugging this into (3.4), for $\delta=\tilde{\delta}(R / \rho)^{-\varepsilon}$ small enough, we arrive at

$$
\int_{B_{\rho}(x)} f^{p} \leq C\left(\frac{R}{\rho}\right)^{(1+p) \varepsilon} \int_{B_{\rho}(x)}|f-(f)|^{p}+C \rho^{n}\left(\frac{R}{\rho}\right)^{(1+p) \varepsilon}\left|(f)^{p}-\left(f^{p}\right)\right| .
$$

Next,

$$
\left|(f)^{p}-\left(f^{p}\right)\right| \leq\left(\left|(f)^{p}-f^{p}\right|\right) \leq\left(|f-(f)|^{p}\right)+\delta f^{p}+\frac{C}{\delta^{p}}\left(|f-(f)|^{p}\right) .
$$


Plugging this now for $\delta=\tilde{\delta}(R / \rho)^{-(1+p) \varepsilon}$ into (3.5), by absorbing we arrive at

$$
\int_{B_{\rho}(x)} f^{p} \leq C\left(\frac{R}{\rho}\right)^{\varepsilon c_{p}} \int_{B_{\rho}(x)}|f-(f)|^{p} .
$$

Since this holds for $\varepsilon>0$ is arbitrarily small, this proves Lemma 1.4.

Corollary 3.1. For $\sigma \in(0,1]$ and for any $f \in W^{\sigma, p}(\Omega)$ satisfying (1.1), $\mathcal{H}^{t}(\Sigma)=$ 0 , whenever $t>s=\max \left\{0, n-\sigma \frac{p \alpha}{p+\alpha}\right\}$.

Proof. Let $\varepsilon>0, R>0$, and $x \in \Sigma$. Pick $\rho<R$ from Lemma 1.4 so that

$$
\int_{B_{\rho}(x)}|f|^{p} \leq C R^{\varepsilon} \rho^{\sigma p-\varepsilon}[f]_{\dot{W}^{\sigma, p}\left(B_{\rho}\right)}^{p} .
$$

By Hölder and Young inequality, as in [8, Corollary 2.1],

$$
\begin{aligned}
\rho^{n+(2 \varepsilon-\sigma p) \frac{\alpha}{p+\alpha}} & \leq C \rho^{2 \varepsilon-\sigma p} \int_{B_{\rho}(x)}|f|^{p}+C \rho^{\varepsilon} \int_{B_{\rho}(x)}|f|^{-\alpha} \\
& \leq C R^{2 \varepsilon}[f]_{W^{\sigma, p}\left(B_{\rho}\right)}^{p}+C R^{\varepsilon} \int_{B_{\rho}(x)}|f|^{-\alpha} .
\end{aligned}
$$

Now let $\varepsilon>0$ such that $t>n+(2 \varepsilon-\sigma p) \frac{\alpha}{p+\alpha}$. Then what we have shown is that for any $R>0$ and any $x \in \Sigma$ there exists $\rho \in(0, R)$ such that

$$
\rho^{t} \leq C R^{\varepsilon}[f]_{W^{\sigma, p}\left(B_{\rho}\right)}^{p}+C \int_{B_{\rho}(x)}|f|^{-\alpha} .
$$

Now let

$$
\mathcal{V}_{R}:=\left\{B_{\rho}(x): x \in \Sigma, \rho<R,(3.6) \text { holds }\right\} .
$$

Any countable disjoint subclass $\mathcal{U}_{R} \subset \mathcal{V}_{R}$ satisfies

$$
\sum_{B_{\rho} \subset \mathcal{U}_{R}} \rho^{t} \leq C R^{\varepsilon}[f]_{\dot{W}^{\sigma, p}(\Omega)}^{p}+C R^{\varepsilon} \int_{\Omega}|f|^{-\alpha} .
$$

By the Besicovitch covering theorem, as in, e.g., [5, Theorem 18.1], we find for any $R$ a countable subclass $\mathcal{U}_{R} \subset \mathcal{V}_{R}$, such that any point of $\Sigma$ is covered at least once, and at most a fixed number of times. Thus,

$$
\mathcal{H}^{t}(\Sigma)=\lim _{R \rightarrow 0} \mathcal{H}_{R}^{t}(\Sigma) \leq C \lim _{R \rightarrow 0} \sum_{B_{\rho} \subset \mathcal{U}_{R}} \rho^{t} \leq C_{f} \lim _{R \rightarrow 0} R^{\varepsilon}=0 .
$$




\section{ACKNOWLEDGMENT}

The author thanks P. Hajłasz for introducing him to Jiang and Lin's paper [8].

\section{REFERENCES}

[1] Robert A. Adams and John J. F. Fournier, Sobolev spaces, 2nd ed., Pure and Applied Mathematics (Amsterdam), vol. 140, Elsevier/Academic Press, Amsterdam, 2003. MR2424078 (2009e:46025)

[2] Xinfu Chen and Huiqiang Jiang, Singular limit of an energy minimizer arising from dewetting thin film model with van der Waal, Born repulsion and surface tension forces, Calc. Var. Partial Differential Equations 44 (2012), no. 1-2, 221-246, DOI 10.1007/s00526-011-0432-9. MR2898777

[3] Juan Dávila and Augusto C. Ponce, Hausdorff dimension of ruptures sets and removable singularities (English, with English and French summaries), C. R. Math. Acad. Sci. Paris 346 (2008), no. 1-2, 27-32, DOI 10.1016/j.crma.2007.11.007. MR2383117 (2008k:35144)

[4] Eleonora Di Nezza, Giampiero Palatucci, and Enrico Valdinoci, Hitchhiker's guide to the fractional Sobolev spaces, Bull. Sci. Math. 136 (2012), no. 5, 521-573, DOI 10.1016/j.bulsci.2011.12.004. MR.2944369

[5] Emmanuele DiBenedetto, Real analysis, Birkhäuser Advanced Texts: Basler Lehrbücher. [Birkhäuser Advanced Texts: Basel Textbooks], Birkhäuser Boston Inc., Boston, MA, 2002. MR.1897317 (2003d:00001)

[6] Zongming Guo and Songbo Hou, Partial regularity for solutions of a nonlinear elliptic equation with singular nonlinearity, J. Math. Anal. Appl. 340 (2008), no. 2, 806-816, DOI 10.1016/j.jmaa.2007.09.002. MR.2390888 (2009f:35086)

[7] Huiqiang Jiang, Energy minimizers of a thin film equation with Born repulsion force, Commun. Pure Appl. Anal. 10 (2011), no. 2, 803-815, DOI 10.3934/cpaa.2011.10.803. MR2754304 (2012b:35099)

[8] Huiqiang Jiang and Fanghua Lin, Zero set of Sobolev functions with negative power of integrability, Chinese Ann. Math. Ser. B 25 (2004), no. 1, 65-72, DOI 10.1142/S0252959904000068. MR:2033951 (2004m:46071)

[9] C. A. Rogers, Hausdorff measures, Cambridge University Press, London, 1970. MR0281862 (43 \#7576)

[10] Stefan G. Samko, Anatoly A. Kilbas, and Oleg I. Marichev, Fractional integrals and derivatives, Gordon and Breach Science Publishers, Yverdon, 1993. Theory and applications; Edited and with a foreword by S. M. Nikol'skiı̌; Translated from the 1987 Russian original; Revised by the authors. MR 1347689 (96d:26012)

Max-Planck Institut MiS Leipzig, Inselstr. 22, 04103 Leipzig, Germany

E-mail address: armin.schikorra@mis.mpg.de 